\title{
Seleção de genótipos de trigo para rendimento de grãos e qualidade de panificação em ensaios multiambientes ${ }^{1}$
}

\author{
Cristiano Lemes da Silva ${ }^{2}$, Elesandro Bornhofen ${ }^{3}$, Matheus Henrique Todeschini $^{4}$, \\ Anderson Simionato Milioli ${ }^{5}$, Diego Maciel Trevizan ${ }^{6}$, Giovani Benin ${ }^{7 *}$ \\ http://dx.doi.org/10.1590/0034-737X201562040005
}

\begin{abstract}
RESUMO
O objetivo deste estudo foi selecionar linhagens de trigo para qualidade de panificação e para rendimento de grãos (RG), em ensaios multiambientes, e identificar ambientes de teste ideais para a seleção. Foram avaliados 39 genótipos de trigo, sendo 18 linhagens e nove cultivares testemunhas, em 2010, e 11 linhagens e quatro cultivares testemunhas, em 2011, no Estado do Paraná. Algumas linhagens e locais não foram repetidos entre os dois anos. O delineamento experimental utilizado foi o de blocos casualizados, com três repetições. A metodologia, em gráfico biplot GGE (Genotype and Genotype-by-Environment), foi utilizada para a avaliação de genótipos e ambientes de teste. Na safra 2010, os locais de Nova Fátima, Pitangueiras e Ventania foram discriminantes (maximizaram a expressão do RG e diferenciação entre genótipos) e representativos do conjunto de ambientes avaliados. Em 2011, Rolândia e Astorga destacaram-se como ambientes ideais para seleção simultânea para RG e qualidade de panificação. As linhagens BIO-08528 e BIO-08228 foram classificadas como genótipos ideais para o RG e a concentração proteica dos grãos (CPG), respectivamente. Na safra 2011, as linhagens BIO-10161 e BIO-10141 foram superiores para RG e para qualidade de panificação, respectivamente. O teste de sedimentação SDS e a CPG correlacionaramse entre si $\left(r=0,61^{* *}\right)$ e foram moderadamente associados com a força de glúten $\left(r=0,49^{* *}\right.$ e $\left.0,74^{* *}\right)$, indicando que podem ser utilizados na seleção indireta para qualidade de panificação.
\end{abstract}

Palavras-chave: Triticum aestivum L., estabilidade, interação genótipo-ambiente, GGE biplot.

\section{ABSTRACT}

\section{Selecting wheat genotypes for yield and baking quality in multi-environment trials}

The objective of this study was to select wheat lines for baking quality and grain yield in multi-environment trials in order to identify ideal test environments for selection. A total of 39 wheat genotypes, 18 inbred lines and 9 checks were evaluated in 2010, and other 11 inbred lines and 4 checks in 2011, in the state of Paraná. Some lines and locations were not repeated between the two years. The experimental design was a randomized block design with three replications. GGE biplot analysis (Genotype and Genotype-by-environment) was used to evaluate the genotypes and test environments. The locations Nova Fátima, Pitangueiras and Ventania were discriminative (maximized the expression of yield potential and the differentiation among genotypes) and representatives of the environments evaluated in the 2010 crop season. Rolândia and Astorga stood out as ideal environments to select

\footnotetext{
Submetido em 26/11/2013 e aprovado em 14/07/2015.

${ }^{1}$ Este Trabalho é parte da dissertação de mestrado do primeiro autor.

Universidade Tecnológica Federal do Paraná, Pato Branco, Paraná, Brasil. cristianolemes.utfpr@gmail.com

${ }^{2}$ Universidade Tecnológica Federal do Paraná, Pato Branco, Paraná, Brasil. elesandro.bornhofen@gmail.com

3 Universidade Tecnológica Federal do Paraná, Pato Branco, Paraná, Brasil. matheus_todeschini@hotmail.com

${ }^{4}$ Universidade Tecnológica Federal do Paraná, Pato Branco, Paraná, Brasil. milioli.utfpr@gmail.com

${ }^{5}$ Universidade Tecnológica Federal do Paraná, Pato Branco, Paraná, Brasil. diegosmi05@ gmail.com

${ }^{6}$ Universidade Tecnológica Federal do Paraná, Pato Branco, Paraná, Brasil. benin @utfpr.edu.br

*Autor para correspondência: benin @utfpr.edu.br
} 
better genotypes for baking quality in 2011. The inbred lines BIO-08528 and BIO-08228 were classified as ideal genotypes for grain yield (GY) and grain protein concentration (GPC), respectively. However, in 2011 the inbred lines BIO-10161 and BIO-10141 were superior for baking quality and GY, respectively. SDS sedimentation test and GPC were correlated each other $\left(\mathrm{r}=0,61^{* *}\right)$ and moderately associated with the gluten strength $\left(\mathrm{r}=0,49\right.$ and $\left.^{* *} 0,74^{* *}\right)$, indicating that both traits can be used for indirect selection of baking quality.

Key words: Triticumaestivum L., stability, genotype-by-environment interaction, GGE biplot.

\section{INTRODUÇÃO}

O Brasil importa aproximadamente metade do trigo que consome, o que se deve, principalmente, a questões econômicas que tornam essa cultura pouco atrativa para os agricultores. Por isso, melhorar a produtividade e a qualidade de panificação é fundamental para tornar a triticultura brasileira mais competitiva. O grande desafio dos melhoristas de trigo consiste em produzir, de forma contínua, novos cultivares que combinem elevada qualidade de panificação e rendimento de grãos, atendendo, simultaneamente, as exigências dos triticultores e do mercado consumidor.

A ocorrência de interação entre genótipo e ambientes $(\mathrm{G} \times \mathrm{A})$ limita a obtenção de ganhos genéticos expressivos e dificulta a seleção e recomendação de cultivares (Ahmadi et al., 2012; Silva \& Benin, 2012). Sendo assim, é necessário realizar avaliações mais precisas, conduzindo ensaios em vários locais e por anos, a fim de identificar genótipos adaptados, estáveis e com características agronômicas adequadas.

Linhagens são testadas, anualmente, em múltiplos locais, por várias razões, entre as quais se destacam: I) identificar genótipos com ampla adaptabilidade com base no desempenho médio em multiambientes; II) selecionar materiais com adaptabilidade específica, com base no desempenho médio em determinados ambientes de um grupo de ambientes. Entretanto, a necessidade de extensiva avaliação em ensaios de competição implica maiores custos de manutenção de uma rede experimental.

Por esta razão, identificar ambientes que facilitem a seleção de genótipos com interação específica contribui para a otimização da rede de ensaios e maximiza a eficiência de seleção (Navabi et al. 2006; Yan et al. 2011; Ahmadi et al., 2012). Embora um ambiente ideal possa não existir, ele pode ser estimado e utilizado como referência para seleção de genótipos em ensaios multiambientes (Kaya et al., 2006), assim como para a escolha de locais com desempenhos próximos ao ideal.
Na presença de interação de natureza complexa, ou seja, quando ocorre inversão no ranqueamento dos genótipos entre os ambientes de teste, a análise GGE (Genotype and Genotype-by-Environment) destaca-se por gerar inferências quanto ao desempenho de genótipos e ambientes, facilitando a visualização dos resultados em saídas gráficas (Silva et al., 2011; Benin et al., 2012; Silva \& Benin, 2012). Além disso, o modelo GGE biplot identifica ambientes representativos e discriminativos e seleciona os genótipos mais adaptados e estáveis a ambientes específicos (Asfaw et al., 2009; Yan, 2010).

O objetivo deste estudo foi identificar linhagens de trigo promissoras e ambientes de testes ideais, que facilitem a seleção de genótipos de trigo com superior rendimento de grãos e qualidade de panificação.

\section{MATERIAL E MÉTODOS}

Os ensaios foram conduzidos nos anos agrícolas de 2010 e 2011, no Estado do Paraná, totalizando 11 locais de cultivo (Tabela 1). Na safra agrícola 2010, 27 genótipos de trigo (18 linhagens e nove cultivares testemunhas) foram testados em nove locais. Já em 2011, 15 genótipos (11 linhagens e quatro cultivares) foram avaliados em quatro locais de cultivo, representativos das regiões de VCU 2 e 3. A rede experimental pertencia ao programa de melhoramento de trigo da empresa Biotrigo Genética.

Os ensaios foram conduzidos em delineamento experimental de blocos ao acaso, com três repetições. Cada parcela constituiu-se de sete linhas de 5,0 metros de comprimento, com espaçamento entre linhas de $0,17 \mathrm{~m}$, formando uma área de $6,0 \mathrm{~m}^{2}$, sendo consideradas como área útil da parcela apenas as cinco linhas centrais. A semeadura foi realizada na primeira e segunda quinzena de maio, nas regiões de VCU2 e VCU3, respectivamente. A densidade de semeadura adotada foi de 350 sementes viáveis por $\mathrm{m}^{2}$.

A adubação de base utilizada foi de $30 \mathrm{~kg} \mathrm{~N} \mathrm{ha}^{-1}, 60$ $\mathrm{kg} \mathrm{P}_{2} \mathrm{O}_{5} \mathrm{ha}^{-1}, 60 \mathrm{~kg} \mathrm{~K}_{2} \mathrm{O} \mathrm{ha}^{-1}$ e mais $70 \mathrm{~kg} \mathrm{ha}^{-1}$ de nitrogê- 
nio na forma de ureia ( $45 \%$ de $N)$, aplicados em cobertura no início do perfilhamento (Z 2.2 da escala Zadocks). Os controles de plantas daninhas, de pragas e de doenças foram realizados de acordo com as recomendações técnicas para a cultura do trigo. O rendimento de grãos (RG) foi determinado a partir do rendimento das parcelas, corrigindo-se para a umidade de $13 \%$ (base úmida) e convertido para $\mathrm{kg} \mathrm{ha}^{-1}$.

Para a análise de qualidade de panificação, o volume de grãos das três repetições de cada genótipo foi misturado e homogeneizado para obtenção de uma amostra composta. As amostras foram analisadas, para qualidade de panificação, no Laboratório de Cereais do Centro de Pesquisa em Alimentação da Universidade de Passo Fundo. As características viscoelásticas da farinha de trigo foram mensuradas em alveógrafo Chopin, modelo NG, conforme a AACC (1995), método $\mathrm{n}^{\circ}$ 54-30. Esse teste avalia a capacidade de absorção de água pelas proteínas formadoras de glúten e a capacidade de retenção do gás carbônico. Entre os parâmetros avaliados na alveografia, apenas a força de glúten (W) foi considerado para análise; esse caractere representa o trabalho mecânico necessário para expandir a massa até a ruptura e é expresso em $10^{-4}$ Joules. A concentração proteica dos grãos (CPG) foi determinada por espectrometria de infravermelho próximo (NIR), com aparelho Infratec Foss 1240, conforme o método no 38-12 da AACC (1995). O volume de sedimentação na presença de SDS (dodecil sulfato de sódio) foi determinado de acordo com o método descrito por Peña \& Amaya (1985). O rendimento de proteína (RP) foi obtido a partir da multiplicação do rendimento de grãos pela concentração proteica dos grãos, de acordo com a expressão: $R P=R G k g h a^{-1} \times C P G(\%)$.

Por causa da prática usual de descarte de linhagens inferiores, e adição de novas nos anos seguintes, grupos diferentes de genótipos foram avaliados em 2010 e 2011. Dessa forma, fez-se necessário analisar individualmente os dados de cada ano.

A análise AMMI1(Additive Main Effects and Multiplicative Interaction) foi utilizada para estimar a contribuição relativa dos efeitos principais de ambiente, genótipo e da interação genótipo $\times$ ambiente. Essa análise considera somente o primeiro eixo de cada componente principal (CP1) e o rendimento de cada genótipo, em cada ambiente $\left(Y_{i j}\right)$, e foi obtida pela equação: $Y_{i j}=Y_{i}+(I P C A 1)_{i}(I P C A)_{j}$, em que $Y_{i}$ é a média do genótipo em todos os ambientes; $I P C A l_{i}$ é a pontuação do genótipo ${ }_{i}$ no primeiro eixo principal da interação IPCA1, estimado pela análise de componentes principais; $I P C A 1_{j}$ é a pontuação do ambiente ${ }_{j}$, sobre o mesmo eixo (IPCA1). Desta forma, o rendimento de cada genótipo em cada ambiente $\left(Y_{i j}\right)$, é representado como uma função linear simples da pontuação ambiental $(I P C A 1)_{\mathrm{j}}$, isto é, uma equação reta de intercepção (IPCA1), (Camargo-Buitrago et al., 2011).

Tabela 1: Identificação das linhagens, cultivares testemunhas e informações de dois locais de ensaios avaliados nas safras agrícolas de 2010 e 2011 nas regiões de Valor de Cultivo e Uso (VCU) 2 e 3

\begin{tabular}{|c|c|c|c|c|c|c|}
\hline Anos & \multicolumn{6}{|c|}{ Genótipos avaliados } \\
\hline 2010 & \multicolumn{6}{|c|}{$\begin{array}{l}\text { 18 linhagens: BIO-06004; BIO-07050; BIO-07219; BIO-07221; BIO-07268; BIO-08023; BIO-08033; BIO-08080; } \\
\text { BIO-08140; BIO-08228; BIO-08264; BIO-08459; BIO-08514; BIO-08527; BIO-08528; } \\
\text { BIO-08537; BIO-08544; BIO-08545. } \\
9 \text { cultivares testemunhas: Mirante; Quartzo; Valente; BRS 208; CD 104; TBIO Bandeirante; TBIO Itaipu; } \\
\text { TBIO Ivaí; TBIO Tibagi. }\end{array}$} \\
\hline 2011 & \multicolumn{6}{|c|}{$\begin{array}{l}11 \text { linhagens: BIO-10115; BIO-10119; BIO-10120; BIO-10121; BIO-10122; BIO-10123; BIO-10141; BIO-10143; } \\
\text { BIO-10152; BIO-10159; BIO-10161. } \\
4 \text { cultivares testemunha: Marfim; Mirante; Quartzo; CD } 104 .\end{array}$} \\
\hline Anos & & Locais & Região de VCU & Latitude & Longitude & Altitude (m) \\
\hline \multirow{7}{*}{2010} & 1 & Cafelândia & 2 & $24^{\circ} 37^{\prime} 4^{\prime \prime} \mathrm{S}$ & $53^{\circ} 19^{\prime} 12^{\prime \prime} \mathrm{W}$ & 521 \\
\hline & 2 & Campo Mourão & 2 & $24^{\circ} 2^{\prime} 45^{\prime \prime} \mathrm{S}$ & $52^{\circ} 22^{\prime} 58^{\prime \prime} \mathrm{W}$ & 630 \\
\hline & 3 & Faxinal & 2 & $24^{\circ} 00^{\prime} 03^{\prime \prime} \mathrm{S}$ & $51^{\circ} 19^{\prime} 12^{\prime \prime} \mathrm{W}$ & 820 \\
\hline & 4 & Ventania & 2 & $24^{\circ} 14^{\prime} 45^{\prime \prime} \mathrm{S}$ & $50^{\circ} 14^{\prime} 34^{\prime \prime} \mathrm{W}$ & 1013 \\
\hline & 5 & Nova Fátima & 3 & $23^{\circ} 25^{\prime} 55^{\prime \prime} \mathrm{S}$ & $50^{\circ} 33^{\prime} 50^{\prime \prime} \mathrm{W}$ & 673 \\
\hline & 6 & Pitangueiras & 3 & $23^{\circ} 13^{\prime} 51^{\prime \prime} \mathrm{S}$ & $51^{\circ} 35^{\prime} 9^{\prime \prime} \mathrm{W}$ & 660 \\
\hline & 7 & Palotina & 3 & $24^{\circ} 17^{\prime} 2^{\prime \prime} \mathrm{S}$ & $53^{\circ} 50^{\prime} 24^{\prime \prime} \mathrm{W}$ & 335 \\
\hline \multirow{4}{*}{2011} & 8 & Apucarana & 3 & $23^{\circ} 33^{\prime} 3^{\prime \prime} \mathrm{S}$ & $51^{\circ} 27^{\prime} 39^{\prime \prime} \mathrm{W}$ & 860 \\
\hline & 9 & Astorga & 3 & $23^{\circ} 13^{\prime} 58^{\prime \prime} \mathrm{S}$ & $51^{\circ} 39^{\prime} 57^{\prime \prime} \mathrm{W}$ & 675 \\
\hline & 10 & Arapongas & 3 & $23^{\circ} 25^{\prime} 8^{\prime \prime} \mathrm{S}$ & $51^{\circ} 25^{\prime} 26^{\prime \prime} \mathrm{W}$ & 816 \\
\hline & 11 & Rolândia & 3 & $23^{\circ} 18^{\prime} 36^{\prime \prime} \mathrm{S}$ & $51^{\circ} 22^{\prime} 08^{\prime \prime} \mathrm{W}$ & 750 \\
\hline
\end{tabular}


A capacidade discriminativa, a representatividade e a associação entre locais de teste foram avaliadas por meio da análise GGE (Genotype and Genotype-byEnvironment). Essa análise considera apenas os efeitos de genótipo $(\mathrm{G})$ e da interação genótipo $\times$ ambiente (G×E) (Yan et al., 2000). A interpretação das associações foi realizada pelo cosseno do ângulo entre vetores, o qual equivale ao coeficiente de correlação. Foram adotados os seguintes critérios: a associação é positiva se o ângulo entre os vetores for $<90^{\circ}$, é negativa se o ângulo for $>90^{\circ}$, e é nula se o ângulo entre os vetores for igual a $90^{\circ}$ (Yan \& Tinker, 2006). A notação Transform $=0$ indica que os dados não foram transformados. No Scaling = 1 (SD), os valores são divididos pelo desvio padrão de cada ambiente, conferindo a cada ambiente importância similar. A notação Centering refere-se ao modelo utilizado, em que Centering $=2$ contém o efeito de GGE (genótipo + interação genótipo $\times$ ambiente). $\mathrm{O}$ SVP, ou "singular value partitioning", é uma técnica matemática para decomposição de matrizes. O SVP = 1, com foco no desempenho genotípico, foi utilizado para a análise da associação entre os caracteres avaliados, e o SVP $=2$, com foco no ambiente, foi utilizado para as demais inferências testadas (Yan et al., 2000). Os coeficientes de correlação de Pearson foram apresentados em forma gráfica, utilizando-se o software Sigmaplot 11.0. As análises em gráfico biplot foram realizadas com o software estatístico GGE biplot (Yan, 2001).

\section{RESULTADOS E DISCUSSÃO}

Os locais de Ventania (7.514 $\left.\mathrm{kg} \mathrm{ha}^{-1}\right)$, Nova Fátima (6.588 $\left.\mathrm{kg} \mathrm{ha}^{-1}\right)$ e Cafelândia $\left(5.371 \mathrm{~kg} \mathrm{ha}^{-1}\right)$ apresentaram rendimentos de grãos (RG) superiores à média geral dos locais dos ensaios conduzidos na safra agrícola de 2010 (Tabela 2). A concentração proteica dos grãos (CPG) foi superior em Astorga, Palotina, Apucarana e Cafelândia. Os maiores valores para o rendimento de proteína (RP) foram observados em Nova Fátima, Ventania, Cafelândia, Apucarana e Astorga. Quanto à relação RG/RP, a qual indica o volume de grãos produzido para se obter um kg de proteína, os menores valores foram verificados em Palotina, Astorga e Apucarana. Na safra agrícola de 2011, Arapongas e Rolândia destacaram-se quanto ao RG (5.295 e $4.950 \mathrm{~kg} \mathrm{ha}^{-1}$, respectivamente) e RP (603 e 592, respectivamente), sendo que Astorga e Apucarana mostraram os menores valores para a relação RG/RP. Estes locais também maximizaram a expressão da maioria dos parâmetros indicativos de qualidade de panificação. Os caracteres força de glúten (W) e CPG tiveram médias de maior magnitude em Apucarana e o caractere SDS apresentou a melhor média em Astorga.
Considerando-se a contribuição relativa da variação proporcionada pelo desdobramento dos efeitos principais de genótipo $(\mathrm{G})$, ambiente (E) e da interação $\mathrm{G} \times \mathrm{E}$, por meio da análise $\mathrm{AMMI}$, verificou-se que o efeito principal de ambiente influenciou em maior magnitude o desempenho dos caracteres avaliados, corroborando os resultados reportados por Asfaw et al. (2009) e Ahmadi et al. (2012). Em 2010, o efeito principal de ambiente explicou 78,92, 70,08, 69,32 e $71,28 \%$ da variação dos componentes RG, CPG, RP e RG/RP, respectivamente, sendo que o efeito de $G$ e da interação $G \times E$ foram relativamente menos importantes (Tabela 2). Da mesma forma, em 2011 o efeito de ambiente foi responsável pela maior variação do caráter rendimento de grãos $(57,91 \%)$, em comparação com o efeito de genótipo $(18,17 \%)$ e com a interação $\mathrm{G} \times \mathrm{E}(13,4 \%)$. Para os demais caracteres, o efeito principal de ambiente representou aproximadamente 13, 45, 11, 45 e $27 \%$ da variação total do RP, RG/RP, SDS, CPG e W, respectivamente. A maior participação do efeito de genótipo em 2011 deve-se à proximidade entre os locais de teste, o que implicou a redução da importância relativa do ambiente sobre os genótipos avaliados.

O genótipo ideal é definido, na análise GGE, com base no alto rendimento de grãos e na estabilidade (Yan \& Kang, 2003). Este "genótipo" é definido graficamente pelo vetor de maior comprimento, no PC1, e sem projeções, em PC2, ou seja, deve estar mais próximo do menor círculo concêntrico central.

Para rendimento de grãos, em 2010, a linhagem BIO 08528 foi a que mais se aproximou do "genótipo idealizado", seguida por BIO 08527, Mirante, Quartzo, TBIO Itaipu, BIO 07221, BIO 08537, BIO 08544 e BIO 08545 (Figura 1), enquanto o cultivar TBIO Ivaí e as linhagens BIO 08264, BIO 08228, BIO 08033, CD 104, BRS 208 e Marfim destacaram-se como os mais próximos ao genótipo ideal para CPG (Figura 1). No ano agrícola de 2011, as linhagens BIO 10161 e BIO 10152 foram as mais próximas do "genótipo ideal" para rendimento de grãos (Figura 2). Da mesma forma, as linhagens BIO 10120, BIO 10122, BIO 10123, BIO 10141 e o cultivar Marfim classificaram-se como mais próximas ao genótipo ideal, para parâmetros indicativos de qualidade de panificação, indicando que reúnem características de elevado rendimento de grãos e qualidade de panificação.

Um ambiente de teste ideal deve discriminar os melhores genótipos e ser representativo de todos os ambientes de teste, ou seja, deve apresentar um alto escore de PC1 (maior poder de discriminação de genótipos) e pontuação nula no PC2 (maior capacidade de representar todos os outros ambientes). Da 
mesma forma, quanto maior o comprimento do vetor de um local de teste, maior a sua capacidade discriminativa (diferenciação de genótipos) e quanto menor o ângulo do vetor de local (em relação ao eixo de seta única), maior a representatividade deste local, em relação ao conjunto de locais avaliados (Yan \& Kang, 2003; Yan \& Tinker, 2006). Ambientes discriminantes e representativos favorecem a seleção de cultivares com adaptação ampla, enquanto ambientes discriminantes, mas não representativos, podem ser úteis para descartar genótipos instáveis, da mesma forma que ambientes não discriminantes e não representativos são pouco úteis em redes experimentais de ensaios e devem ser descartados.

Tabela 2: Médias $^{2}$, amplitude de variação ${ }^{3}$ e contribuição relativa dos efeitos principais (EP) ${ }^{4}$ de Genótipo (G), Ambiente (E) e interação (G×E), com base na análise AMMI1, de quatro caracteres agronômicos mensurados em 28 genótipos de trigo avaliados nas regiões de VCU 2 e 3 em 2010 e seis caracteres mensurados em 15 genótipos de trigo avaliados na região de VCU 3 na safra 2011

\begin{tabular}{|c|c|c|c|c|c|c|}
\hline \multicolumn{7}{|c|}{2010} \\
\hline Ambientes & \multicolumn{2}{|r|}{$\mathbf{R G}^{\mathbf{1}}$} & \multicolumn{2}{|l|}{ CPG } & $\mathbf{R P}$ & RG/RP \\
\hline \multirow[t]{2}{*}{ Cafelândia } & \multicolumn{2}{|r|}{$5371^{2}$} & 11,3 & \multicolumn{2}{|r|}{608} & 8,2 \\
\hline & \multicolumn{2}{|r|}{$4389-5752^{3}$} & $11,1-12,7$ & \multicolumn{2}{|r|}{$504,6-672$} & $7,9-9,0$ \\
\hline \multirow[t]{2}{*}{ Campo Mourão } & \multicolumn{2}{|r|}{4418} & 11,0 & \multicolumn{2}{|r|}{486} & 9,08 \\
\hline & \multicolumn{2}{|r|}{$2988-4943$} & $10,2-13,5$ & \multicolumn{2}{|r|}{$396,2-562,6$} & $7,4-9,8$ \\
\hline \multirow[t]{2}{*}{ Faxinal } & \multicolumn{2}{|r|}{4358} & 10,5 & \multicolumn{2}{|r|}{456} & 9,55 \\
\hline & \multicolumn{2}{|r|}{$3056-4732$} & $9,9-12,4$ & \multicolumn{2}{|r|}{$329,5-503,5$} & $8,1-10,1$ \\
\hline \multirow[t]{2}{*}{ Ventania } & \multicolumn{2}{|r|}{7514} & 10,3 & \multicolumn{2}{|r|}{774} & 9,7 \\
\hline & \multicolumn{2}{|r|}{$5081-7783$} & $9,7-10,8$ & \multicolumn{2}{|r|}{$500,6-809,4$} & $9,2-10,3$ \\
\hline \multirow[t]{2}{*}{ Nova Fátima } & & 6588 & 11,3 & & 747 & 8,82 \\
\hline & & $4433-7429$ & $9,7-12,2$ & & $513,3-792,1$ & $8,2-10,4$ \\
\hline Apucarana & & 4931 & 12,7 & & 628 & 7,84 \\
\hline & & $3858-5706$ & $10,9-13,8$ & & $437,6-681,2$ & $7,2-9,2$ \\
\hline Pitangueiras & & 4951 & 11,0 & & 543 & 9,1 \\
\hline & & $3250-5189$ & $10,8-13,1$ & & $385,5-568,8$ & $7,6-9,2$ \\
\hline Astorga & & 4426 & 12,9 & & 570 & 7,76 \\
\hline & & $3817-5111$ & $12,5-15,1$ & & $504,7-638,9$ & $6,6-8,0$ \\
\hline Palotina & & 4646 & 13,0 & & 604 & 7,69 \\
\hline & & $3341-5086$ & $11,6-14$ & & $428,4-630,3$ & $7,1-8,6$ \\
\hline Médias & & 5244,77 & 11,55 & & 601,77 & 8,63 \\
\hline Fontes de variação & & Contri & relativa dos e & eitos princip & pais pela análise $\mathrm{AN}$ & \\
\hline EP Ambiente (E) & & 78,92 & 70,08 & & 69,32 & 71,28 \\
\hline EP Genótipo $(\mathrm{G})$ & & 9,23 & 16,25 & & 10,5 & 14,40 \\
\hline EP interação (G×E) & & 4,10 & 4,20 & & 9,20 & 4,60 \\
\hline & & & 2011 & & & \\
\hline Ambientes & RG & $\mathbf{R P}$ & RG/RP & SDS & CPG & $\mathbf{W}$ \\
\hline Astorga & 4654,0 & 590,0 & 7,9 & 68,0 & 12,7 & 314,0 \\
\hline & $4040-5492$ & $508-671$ & $7,2-8,7$ & $53,5-81,5$ & $11,5-13,9$ & $232-420$ \\
\hline Apucarana & 4286,0 & 553,0 & 7,8 & 66,5 & 12,9 & 345,0 \\
\hline & $3588-4795$ & $426-674$ & $7,1-8,5$ & $49-80$ & $11,7-14,3$ & $224-468$ \\
\hline Rolândia & 4950,0 & 592,0 & 8,4 & 61,1 & 12,0 & 257,0 \\
\hline & $4631-5814$ & $532-724$ & $7,75-8,97$ & $53,5-77$ & $10,6-12,9$ & $195-344$ \\
\hline Arapongas & 5295,0 & 603,0 & 8,8 & - & 11,4 & 309,0 \\
\hline & $4795-5770$ & $563-638$ & $8,2-9,3$ & - & $10,8-12,2$ & $208-384$ \\
\hline Médias & 4796,25 & 584,5 & 8,225 & 65,2 & 12,25 & 306,25 \\
\hline Fontes de variação & & & buição relativa & dos efeitos $p$ & principais $^{4}$ & \\
\hline EPAmbiente (E) & 57,91 & 13,11 & 45,55 & 11,26 & 44,72 & 26,94 \\
\hline EP Genótipo (G) & 18,17 & 51,55 & 48,41 & 76,97 & 47,64 & 50,85 \\
\hline EP interação $(\mathrm{G} \times \mathrm{E})$ & 13,40 & 21,90 & 3,80 & 9,40 & 5,40 & 11,50 \\
\hline
\end{tabular}

${ }^{1}$ RG: Rendimento de Grão(kg ha-1); CGP: Concentração Proteica dos Grãos (\%); RP: Rendimento de Proteína (kg ha ${ }^{-1}$ ); SDS: Sedimentação; W: Força de glúten (10 $10^{-4}$ Joules); ${ }^{4}$ Contribuição dos efeitos de ambiente (E), genótipos (G) e interação (G×E) mediante análise AMMI1 (Additive Main Effects and Multiplicative Interaction).

Rev. Ceres, Viçosa, v. 62, n.4, p. 360-371, jul-ago, 2015 
No ano de 2010, os locais de Pitangueiras, Nova Fátima e Ventania foram classificados como ambientes ideais de teste para rendimento de grãos e, além disso, foram simultaneamente os ambientes mais discriminantes e representativos (Figura 3). Provavelmente isso se deve à condição de irrigação (pivô central), em Pitangueiras e em Nova Fátima, e aos elevados níveis de fertilidade, em Ventania, que maximizaram a expressão do potencial de rendimento dos genótipos. Ventania mostrou-se o local menos discriminativo para CPG e para a relação RG/RP e os locais de Campo Mourão e Faxinal apresentaram informações redundantes para rendimento de grãos. A identificação e o descarte de locais redundantes reduzem os custos de pesquisa com avaliações de campo e mão de obra, sem perda de informação genotípica (Pacheco et al., 2009; Zhe et al., 2010). Cafelândia, Faxinal e Apucarana foram classificados como ambientes ideais para avaliar CPG; porém, com alta relação $\mathrm{RG} / \mathrm{RP}$, por causa do baixo rendimento de grãos (RG) obtido nesses locais, uma vez que a CPG e o RG são inversamente associados (Bogard et al., 2010; Gaju et al., 2011). Assim, fica evidente a necessidade de se identificarem genótipos que maximizem o aproveitamento de estímulos específicos de ambiente, a fim de incrementar o RG e a qualidade de panificação, simultaneamente.

Na safra agrícola de 2011, Rolândia classificou-se como ambiente ideal (representativo e discriminante) para RG e CPG, enquanto Apucarana destacou-se como ambiente ideal para os parâmetros indicativos de qua-
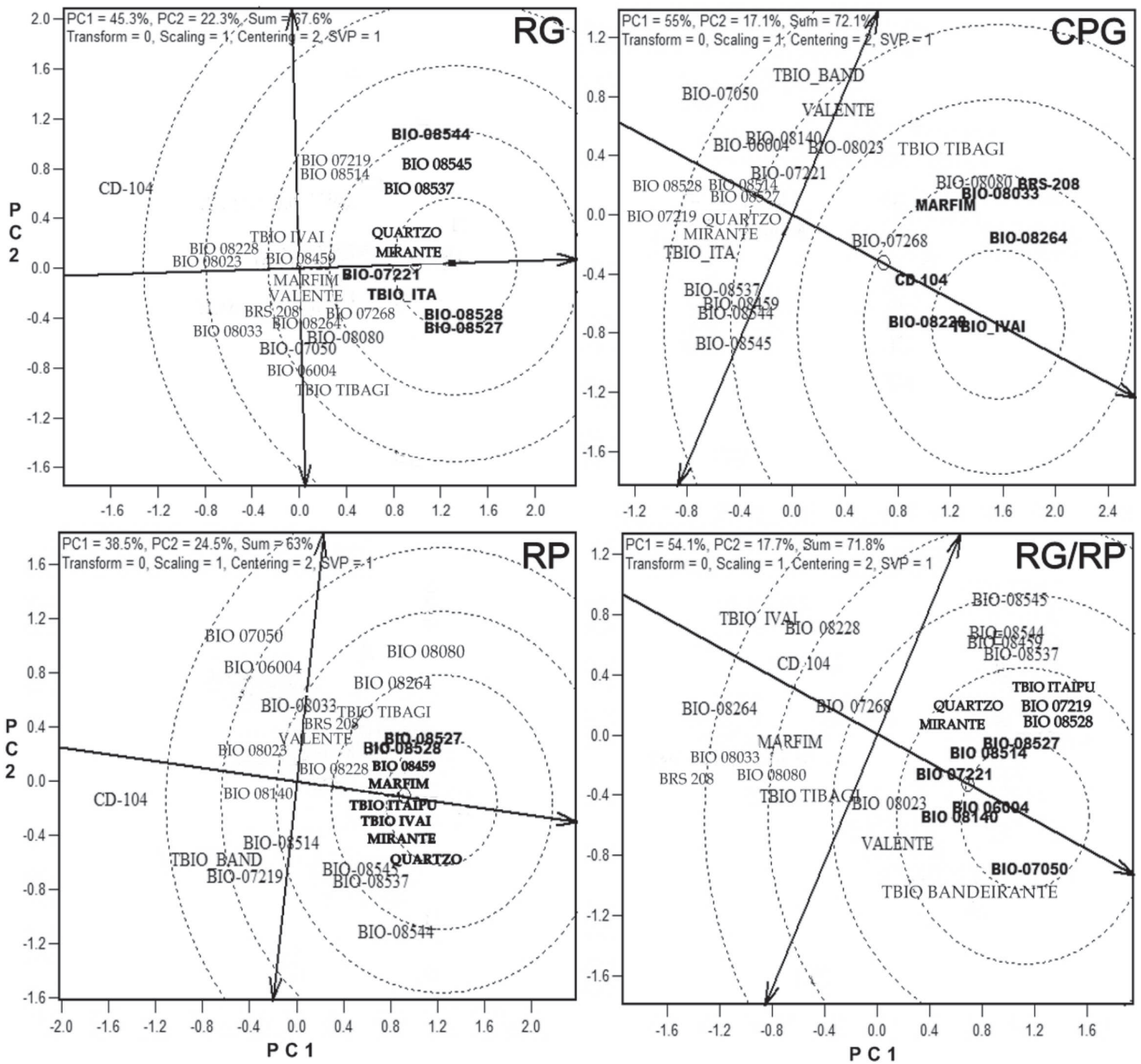

Figura 1: Plotagem dos escores dos componentes principais, segundo o modelo GGE biplot, quanto a identificação do genótipo ideal para os caracteres rendimento de grãos (RG), concentração proteica dos grãos (CPG), rendimento de proteína (RP) e relação RG/RP de 28 genótipos de trigo avaliados em 9 locais de ensaio, na safra 2010. 
lidade de panificação SDS e W (Figura 4). Arapongas destacou-se como o ambiente menos representativo para o conjunto de caracteres avaliados, sendo útil apenas para a seleção de genótipos especificamente adaptados. Por terem sido testados apenas quatro locais, em 2011, não foi possível identificar ambientes redundantes.

As associações entre os caracteres avaliados com os genótipos e os locais de cultivo são apresentadas na Figura 5. O cosseno de um ângulo entre os vetores de
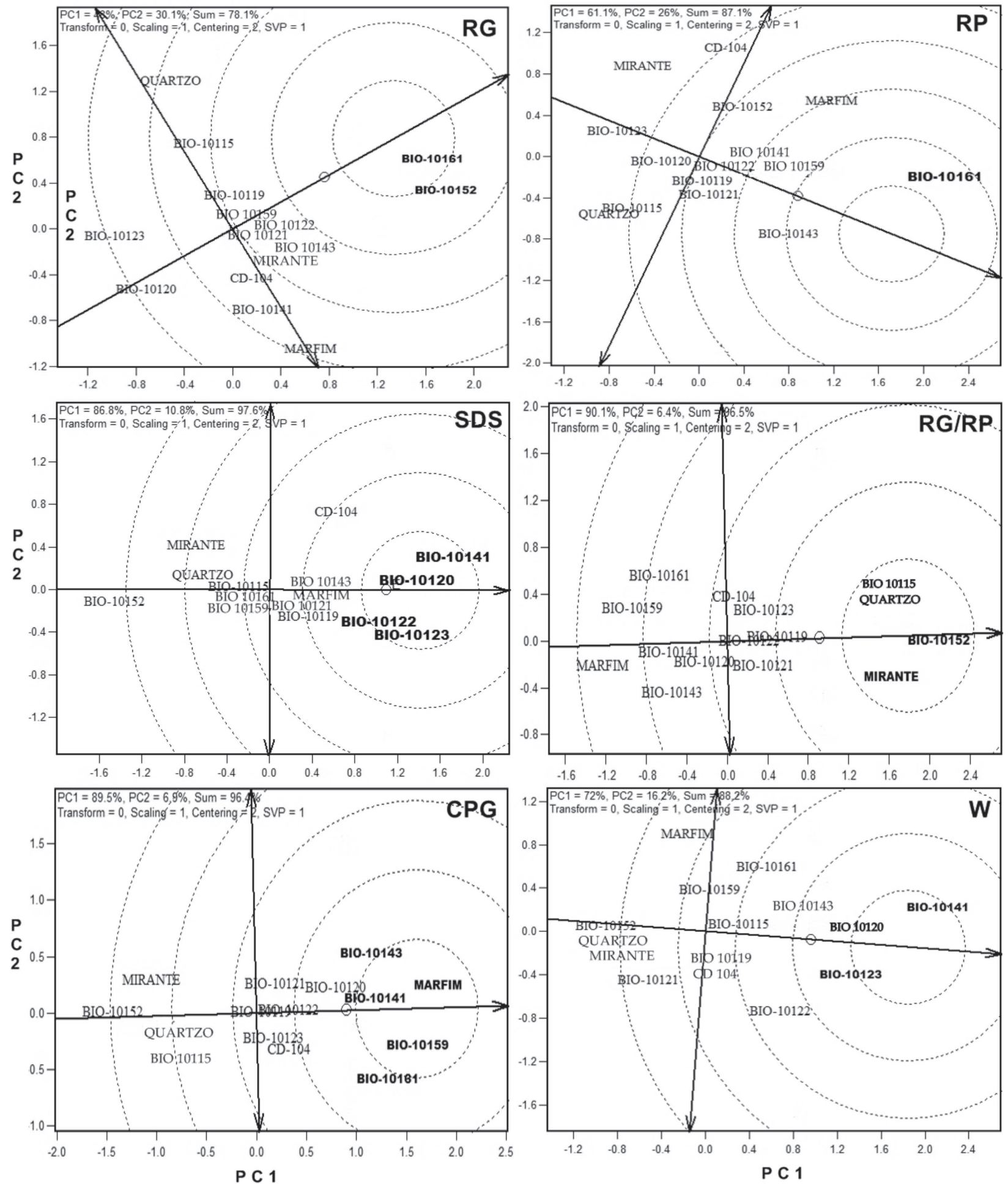

Figura 2: Plotagem dos escores dos componentes principais, segundo o modelo GGE biplot, quanto à identificação do genótipo ideal para os caracteres rendimento de grãos (RG), rendimento de proteína (RP), teste de sedimentação em SDS (SDS), relação RG/RP, concentração proteica dos grãos (CPG) e forca de glúten (W) de 15 genótipos de trigo avaliados em 4 locais da região de VCU3 no Estado do Paraná, na safra 2011.

Rev. Ceres, Viçosa, v. 62, n.4, p. 360-371, jul-ago, 2015 
quaisquer dois caracteres representa a correlação entre esses caracteres, sendo que quanto menor for o ângulo entre dois vetores, maior é a correlação entre eles (Yan \& Kang, 2003). Na safra 2010, a CPG teve sua expressão maximizada (foi positivamente associada) nas condições ambientais de Palotina, Astorga e Apucarana (Figura 5a), com destaque para o cultivar Marfim e para a linhagem BIO 08264 (Figura 5b). Da mesma forma, Nova Fátima e Ventania associaram-se positivamente com os caracteres RG e RP (Figura 5a), com destaque para as linhagens BIO 08528, BIO08527 e BIO 08544 (Figura $5 b)$. Inferências a cerca do rendimento de grãos podem ser extraídas de apenas um desses locais, em vista da associação positiva existente, conduzindo a informações seguras do conjunto de genótipos avaliados. Kaya et al. (2006) e Jalata (2011) salientam que pode ser aplicada seleção indireta entre ambientes de testes correlacionados, obtendo-se o mesmo nível de informação de ambi- ente e economia, com a otimização do número de ensaios multiambientes.

Em 2010, os desempenhos dos parâmetros indicativos de qualidade de panificação CPG, SDS e W foram maximizados, nos locais de Astorga e Apucarana (Figura 5c), com destaque para as linhagens BIO10141 e BIO10120 (Figura 5d). Em 2011, os caracteres RG e CPG foram negativamente correlacionados $(\mathrm{r}=-0,53 * *)$ (Figura 6a), provavelmente por causa da competição entre carbono e nitrogênio por energia (Munier-Jolain et al., 2005; Xu et al., 2012). Outros estudos também confirmam esta relação negativa entre RG e CPG (Bogard et al., 2010; Gaju et al., 2011). Entretanto, associação positiva e de elevada magnitude foi evidenciada entre os caracteres RG e RP (0,89**) (Figura 6b). Embora ocorra redução da CPG em detrimento da elevação no RG, há maior rendimento de proteína associado com o aumento do RG, quando a observação é ex-
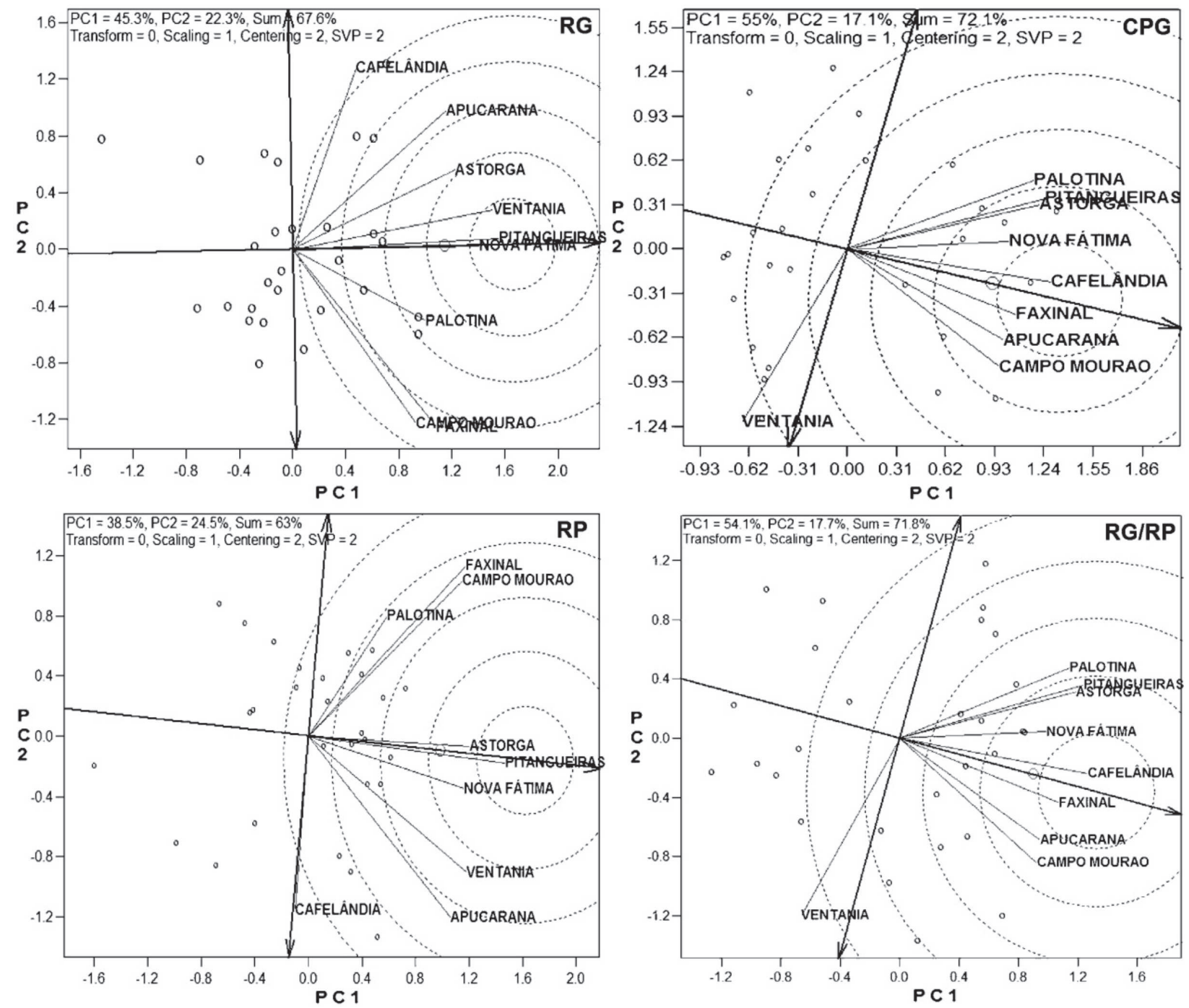

Figura 3: Plotagem dos escores dos componentes principais, segundo o modelo GGE biplot, quanto a identificação de ambiente ideal para os caracteres rendimento de grãos (RG), concentração proteica dos grãos (CPG), rendimento de proteína (RP) e relação RG/RP de 28 genótipos de trigo avaliados em 9 locais de ensaio, na safra 2010. 
traída de uma unidade de área, pois ocorre uma compensação do menor valor de CPG, em virtude do maior volume de grãos.

A força de glúten (W) foi positivamente correlacionada com a CPG $(\mathrm{r}=0,49 * *)($ Figura $6 \mathrm{c})$ e com o teste de sedimentação em $\operatorname{SDS}(\mathrm{r}=0,74 * *)$ (Figura 6d), corroborando outros estudos similares (Clarke et al., 2010; Li et al., 2013). A CPG e o SDS $(\mathrm{r}=0,61 * *)$ também foram positivamente associados entre si (Figura 6e). Esses resultados indicam
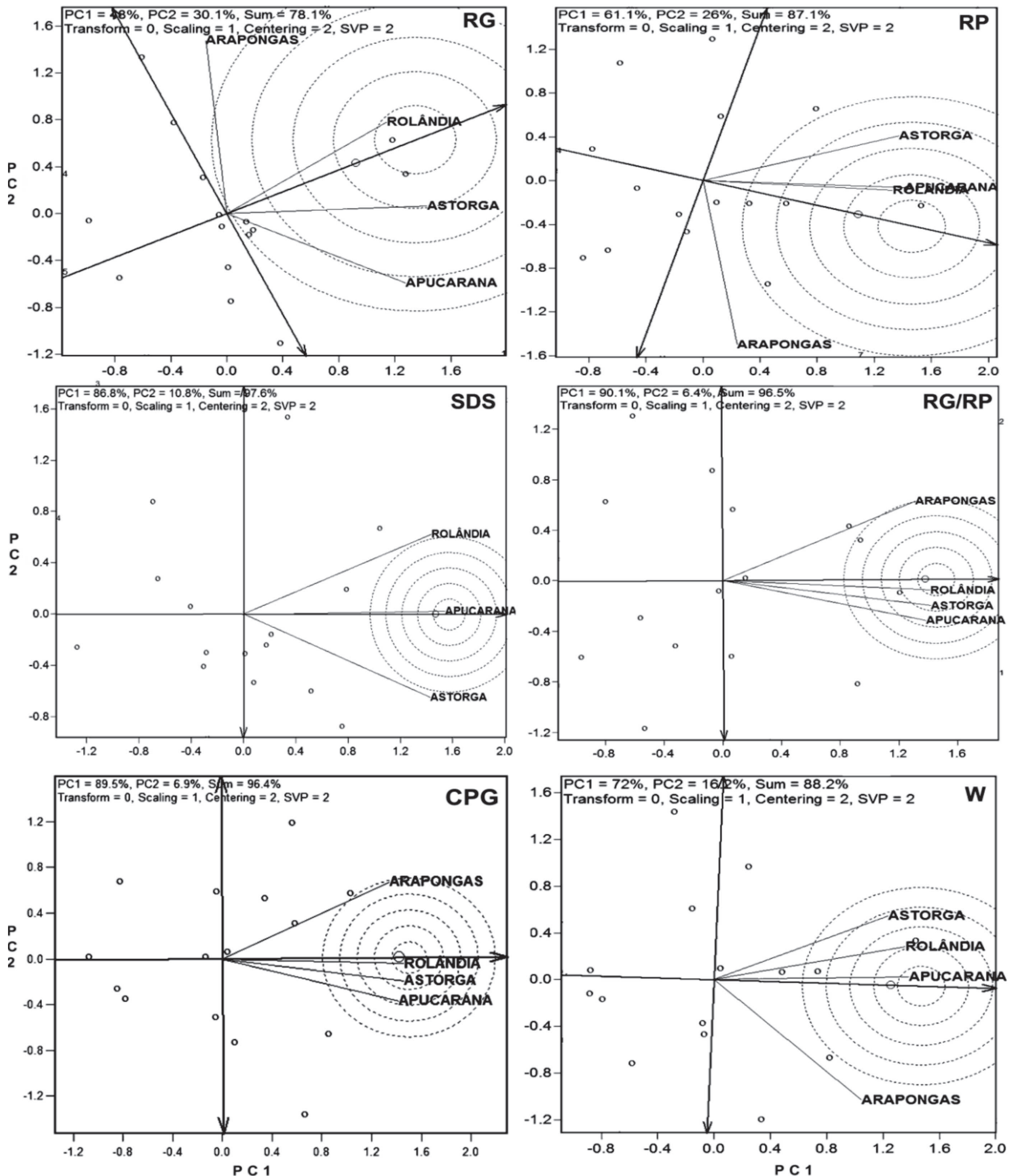

Figura 4: Plotagem dos escores dos componentes principais, segundo o modelo GGE biplot, quanto a identificação de ambiente ideal para os caracteres rendimento de grãos (RG), rendimento de proteína (RP), teste de sedimentação em SDS (SDS), relação RG/RP, concentração proteica dos grãos (CPG) e forca de glúten (W) de 15 genótipos de trigo avaliados em 4 locais da região de VCU3 no Estado do Paraná, na safra 2011.

Rev. Ceres, Viçosa, v. 62, n.4, p. 360-371, jul-ago, 2015 
que tanto a CPG como o SDS podem ser usados de forma eficiente na seleção indireta para estimar valores de W (Al-Saleh e Brennan, 2012). Clarke et al (2010) observaram valores de correlação de 0,62 a 0,96, entre SDS e W, e de 0,52, entre SDS e CPG, e sugeriram que o teste de sedimentação SDS pode ser usado com eficácia na seleção de genótipos de trigo com elevada qualidade de panificação, pois esse teste exige baixo volume de amostra, menor necessidade de mão de obra e é relativamente barato, em comparação com as análises convencionais de W e CPG. Segundo Quick \& Donnelly (1980), a seleção para força de glúten com base no teste SDS pode ser $84 \%$ bem sucedida, em relação às análises tradicionais de qualidade de panificação. Além disso, um laboratorista bem treinado consegue realizar mais de 200 análises diárias.
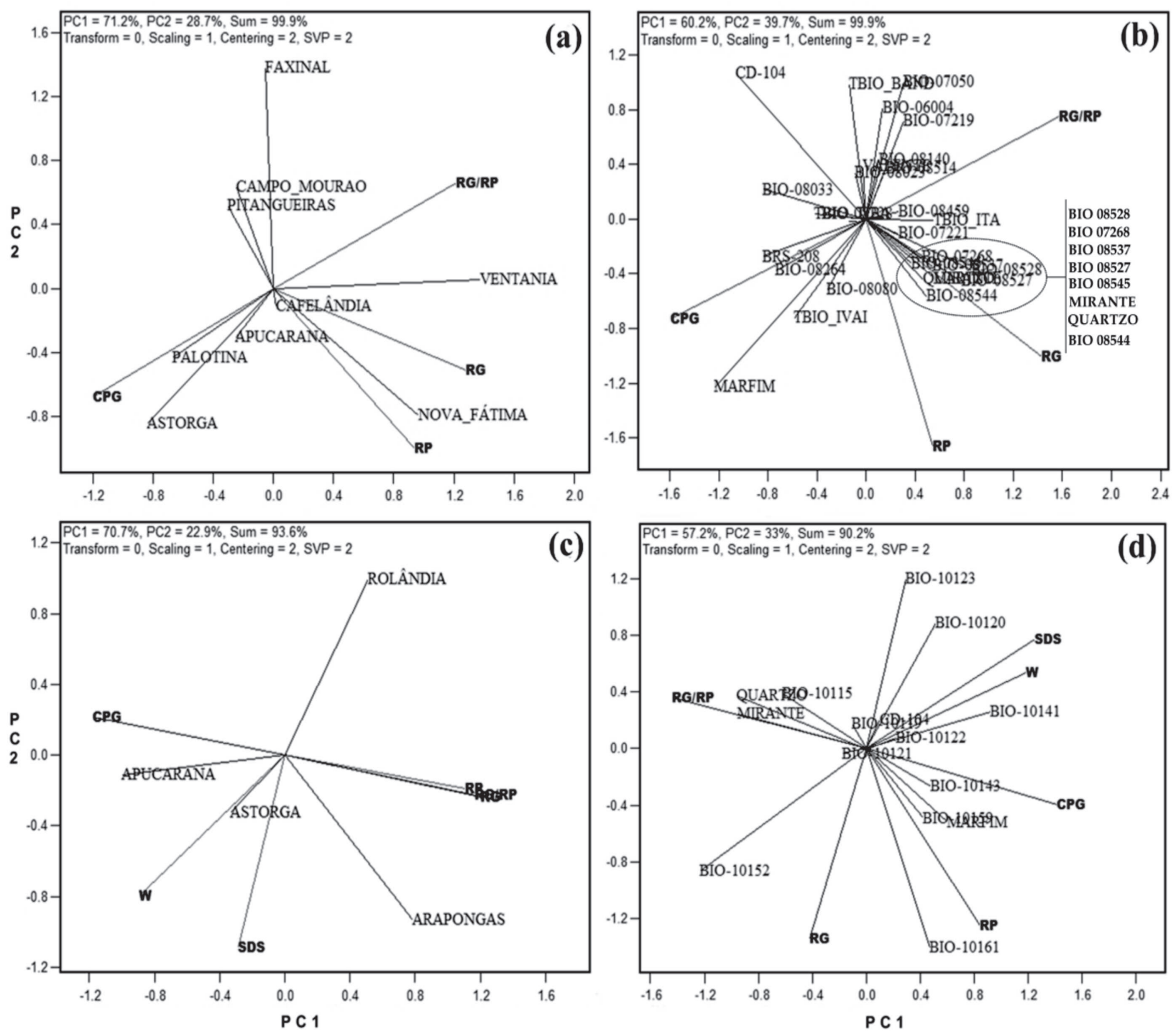

Figura 5: Plotagem dos escores dos componentes principais, segundo o modelo GGE biplot, quanto a associação entre 4 caracteres agronômicos e 9 locais de ensaios de trigo (a) e entre caracteres e genótipos (b), na safra 2010 e associação entre 8 caracteres agronômicos e 4 locais de ensaios de trigo (c) e entre caracteres e genótipos (d), na safra 2011. 

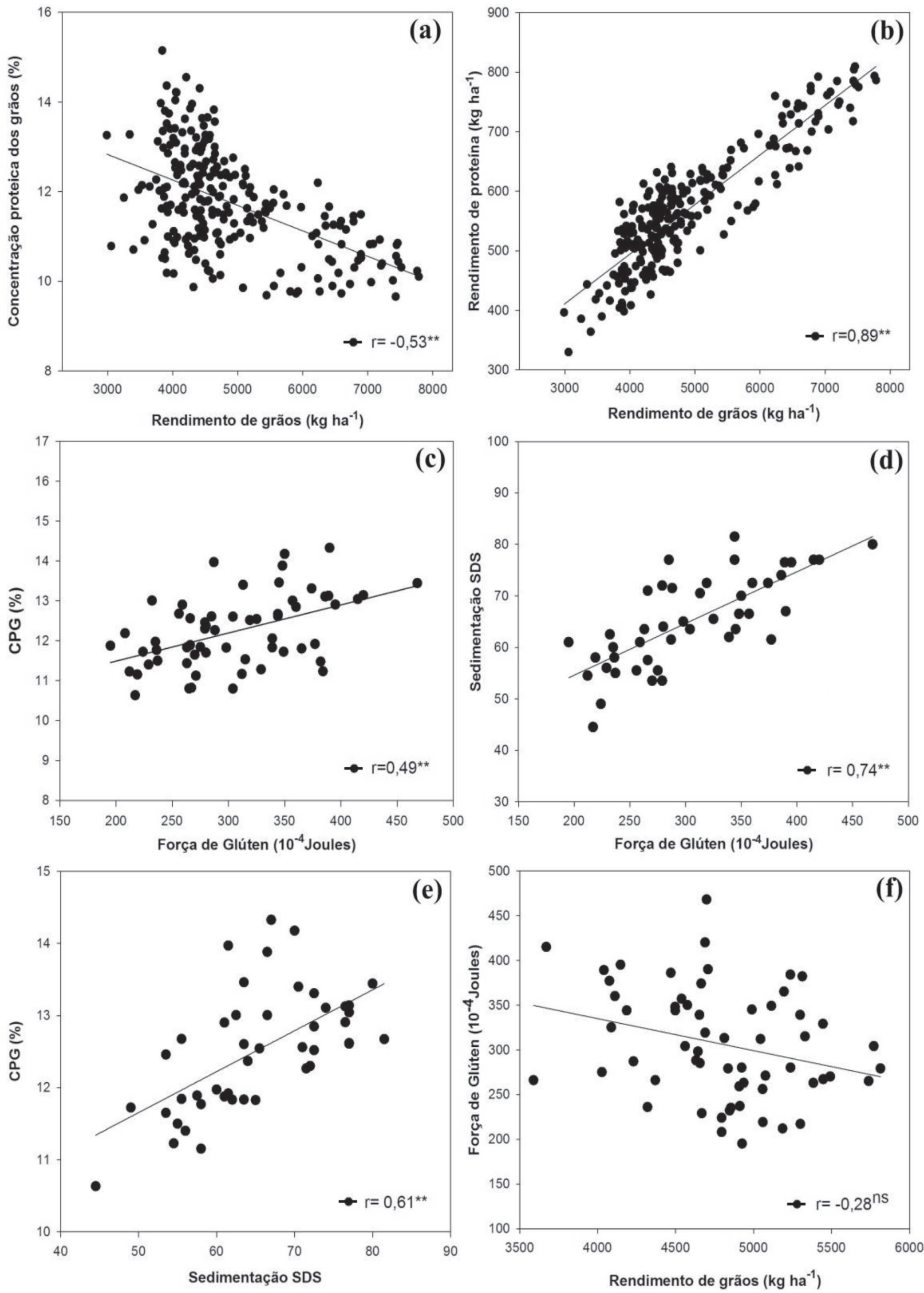

Figura 6: Estimativas de correlação de Pearson entre 3 caracteres agronômicos avaliados em 27 genótipos de trigo conduzidos em 9 locais de testes no Estado do Paraná, na safra 2010 (a; b) e correlação entre 4 caracteres agronômicos avaliados em 14 genótipos de trigo conduzidos em 4 locais de teste no Estado do Paraná, safra 2011 (c; d; e; f). 


\section{CONCLUSÕES}

As linhagens BIO-08228, BIO-10161, BIO-08528 e BIO-10141 destacaram-se simultaneamente como genótipos ideais para o rendimento de grãos e a qualidade de panificação.

Os locais de teste Pitangueiras, Nova Fátima, Rolândia e Astorga foram ambientes representativos e discriminantes do rendimento de grãos e de parâmetros indicativos da qualidade de panificação.

A seleção para qualidade de panificação pode ser feita indiretamente, por meio da concentração proteica dos grãos ou teste de sedimentação em SDS.

\section{REFERÊNCIAS}

AACC - American Association of Cereal Chemists (1995) Approved methods. $9^{\text {a }}$ ed. Saint Paul, AACC.

Ahmadi J, Mohammadi A \& Mirak N (2012) Targeting promising bread wheat (Triticumaestivum L.) lines for cold climate growing environments using AMMI and SREG GGE Biplot analyses. Journal of Agricultural Science and Technology, 14:645-657.

Al-Saleh A \& Brenna CS (2012) Bread wheat quality: some physical, chemical and rheological characteristics of Syrian and English bread wheat samples. Foods, 1:03-17.

Asfaw A, Alemayehu F, Gurum F \& Atnaf M (2009) AMMI and GGE Biplot analysis for matching varieties onto soybean production environments in Ethiopia. Scientific Researchand Essay, 4:13221330.

Benin G, Pinnow C, Silva CL, Pagliosa ES, Beche E, Bornhofen E, Munaro LB \& Silva RR (2012) Análises biplot na avaliação de cultivares de trigo em diferentes níveis de manejo. Bragantia, 71:28-36.

Bogard M, Allard V, Brancourt-Hulmel M, Heumez E, Machet JM, Jeuffroy MH, Gate P, Martre P \& Le Gouis J (2010) Deviation from the grain protein concentration-grain yield negative relationship is highly correlated to post-anthesis $\mathrm{N}$ uptake in winter wheat. Journal of Experimental Botany, 61:4303-4312.

Camargo-Buitrago I, Intire EQ \& Gordón-Mendoza R (2011) Identificación de mega-ambientes para potenciar el uso de genotipos superiores de arroz em Panamá. Pesquisa Agropecuária Brasileira, 46:1061-1069.

Clarke FR, Clarke JM, Ames NA, Knox RE \& Ross JR (2010) Gluten index compared with SDS-sedimentation volume for early generation selection for gluten strength in durum wheat. Canadian Journal of Plant Science, 90:01-11.

Gaju O, Allard V, Martre P, Snape JW, Heumez E, Le Gouis J, Moreau D, Grifiths S, Orford S, Hubbart S, Foulkes MJ \& Bogard M (2011) Identiûcation of traits to improve the nitrogen-use efûciency of wheat genotypes. Field Crops Research, 123:139-152.

Jalata Z (2011) GGE-biplot Analysis of Multi-environment Yield Trials of Barley (Hordeiumvulgare L.) Genotypes in Southeastern Ethiopia Highlands. International Journal of Plant Breeding and Genetics, 5:5975 .

Kaya Y, Akçura M \& Taner S (2006) GGE-Biplot Analysis of MultiEnvironment Yield Trials in Bread Wheat. Turkish Journal of Agriculture and Forestry, 30:325-337.

Li Y, Wu Y, Espinosa NH \& Peña RJ (2013) The influence of drought and heat stress on the expression of end-use quality parameters of common wheat. Journal of Cereal Science, 57:73-78.
Munier-Jolain NG \& Salon C (2005) Are the carbon costs of seed production related to the quantitative and qualitative performance? An appraisal for legumes and other crops. Plant, Cell and Environment, 28:1388-1395.

Navabi A, Yanga RC, Helmb J \& Spaner DM (2006) Can Spring WheatGrowing Mega environments in the Northern Great Plains Be Dissected for Representative Locations or Niche-Adapted Genotypes? Crop Science, 46:1107-1116.

Pacheco RM, Duarte JB, Souza PIM, Silva AS \& Nunes Junior J (2009) Key locations for soybean genotype assessment in Central Brazil. Pesquisa Agropecuaria Brasileira, 44:478-486.

Peña RJ \& Amaya A (1985) Rapid estimation of gluten quality in bread wheat, durum wheat and triticale using the sodium dodecyl sulfate (SDS) - sedimentation test: grain quality methods. Mexico, International Maize and Wheat Improvement Center. 12p.

Quick JS \& Donnelly BJ (1980) A rapid test for estimating durum wheat gluten quality. Crop Science, 20:816-818.

Silva RR \& Benin (2012) G Análises Biplot: conceitos, interpretações e aplicações. Ciência Rural, 42:1404-1412.

Silva RR, Benin G, Silva GO, Marchioro VS, Almeida JL \& Matei G (2011) Adaptabilidade e estabilidade de cultivares de trigo em diferentes épocas de semeadura, no Paraná. Pesquisa Agropecuária Brasileira, 46:1439-1447.

Yan W, Pageau D, Frégeau-Reid JA \& Durand J (2011) Assessing the representativeness and repeatability of test locations for genotype evaluation. Crop Science, 51:1603-1610.

Yan W (2001) GGE biplot - A Windows application for graphical analysis of multi-environment trial data and other types of two-way data. Agronomy Journal, 93:1111-1118.

Yan W (2010) Optimal use of biplots in analysis of multi-location variety test data. Acta Agronomica Sinica, 36:1805-1819.

Yan W \& Kang MS (2003) GGE biplot analysis: A graphical tool for breeders, geneticists, and agronomists. $1^{\mathrm{a}}$ ed. Florida, CRC Press. $288 \mathrm{p}$.

Yan WK, Hunt LA, Sheng QL \& Szlavnics Z (2000) Cultivar evaluation and mega-environment investigation based on the GGE biplot. Crop Science, 40:597-605.

Yan W \& Tinker A (2006) Biplot analysis of multi environment trial data: principles and applications. Canadian Journal of Plant Science, 86:623645.

Xu G, Fan X \& Miller AJ (2012) Plant Nitrogen Assimilation and Use Efficiency. Annual Review of Plant Biology, 63:153-82.

Zhe Y, Lauer JG, Borges R \& Leon N (2010) Effects of genotype $\times$ environment interaction on agronomic traits in soybean. Crop Science, 50:696-702. 